\title{
Design and Development of Dentistry Ethical Codes: Proposed for National Implementation in Iran
}

\section{Azam Khorshidian', Bagher Larijani',3, Mohammad Sadegh Ahmad- Akhoundi $^{4}$, Alireza Parsapour ${ }^{1,3}$, Abbas Ebadi ${ }^{5,6}$, Amir Ahmad Shojaei ${ }^{1,3^{*}}$}

1. Department of Medical Ethics, Faculty of Medicine, Tehran, University of Medical Sciences, Tehran, Iran

2. Endocrinology and Metabolism Research Center, Endocrinology and Metabolism Clinical Sciences Institute, Tehran University of Medical Sciences, Tehran, Iran

3. Medical Ethics and History of Medicine Research Center, Tehran University of Medical Sciences, Tehran, Iran

4. Dental Research Center, Dentistry Research Institute, Tehran University of Medical Sciences, Tehran, Iran

5. Behavioral Sciences Research Center, Life Style Institute, Baqiyatallah University of Medical Sciences, Tehran, Iran

6. Nursing Faculty, Baqiyatallah University of Medical Sciences, Tehran, Iran

\begin{tabular}{ll}
\hline Article Info & A B S T R A C T \\
\hline $\begin{array}{l}\text { Article type: } \\
\text { Original Article }\end{array}$ & $\begin{array}{l}\text { Objectives: The dental profession has a special place of trust in the society, and dental } \\
\text { clinicians must adhere to ethical standards in all dental procedures. Ethical conduct is } \\
\text { one of the main expectations of individuals from this profession. The aim of this study } \\
\text { was to design and develop dental ethical codes for national implementation in Iran. }\end{array}$
\end{tabular}

\section{Article history:}

Received: 16 March 2018

Accepted: 28 September 2018

Published: 30 August 2019
Materials and Methods: This qualitative study was performed using directed content analysis method and purposive sampling. Data were collected until saturation through 15 semi-structured face-to-face individual interviews and two expert panels with academic staffs from dental faculties in Tehran, Iran.

Results: Data were classified into five principles and 90 codes. The principles included consideration of patients' interest as a priority, respect for human dignity and patient autonomy, confidentiality of patient information, the excellence of knowledge and skills, and building trust.

* Corresponding author:

Department of Medical Ethics, Faculty of Medicine, Tehran, University of Medical Sciences, Tehran, Iran

Email: dramirahmadsh@yahoo.com
Conclusion: The ethical codes for Iranian dentists were drafted considering Islamic teachings and the prevailing culture. Some codes were exclusively developed for the cultural atmosphere of Iran especially on topics such as interaction with patients previously treated by other dentists. Some codes addressed the principles of consultation and continuing the therapeutic communication with such patients. Some items have not been considered in codes released by other associations, such as religious considerations in Islamic cover and alcohol consumption that were taken into consideration in this draft. These codes can serve as a guide for professional practice of dentists. It seems that these sets can help us reach the standardized code.

Keywords: Codes of Ethics; Dentistry; Health Services Administration; Patient Rights

> Cite this article as: Khorshidian A, Larijani B, Ahmad-Akhoundi MS, Parsapour A, Ebadi A, Shojaei AA. Design and Development of Dentistry Ethical Codes: Proposed for National Implementation in Iran. Ceramic. Front Dent. 2019;16(4):303-318. doi:

This work is published as an open access article distributed under the terms of the Creative Commons Attribution 4.0 License (http://creativecommons.org/licenses/by-nc/4). Non-commercial uses of the work are permitted, provided the original work is properly cited. 


\section{INTRODUCTION}

The mission of dentistry is to provide highquality services to preserve and promote the oral and dental health of the population. These preventive and therapeutic services should be delivered in an appropriate manner with acceptable standards and up-to-date knowledge [1]. The principles of professional ethics comprise the commitments that people in a profession have towards the society. The society expects a professional to give priority to patients and public health over personal benefits in all professional decisions, which is the most important way of enhancing the public trust [2]. To provide dental services with maximum efficacy, it is necessary that service providers commit to advocate ethical considerations, which would result in the peace of mind and security of patients and the society.

Medical ethics creates a specific attitude aiming at improving the quality of patient care by identifying, analyzing, and solving ethical problems that arise in practice [3]. Ethical goals and values, formulated as ethical codes, can be utilized as a source for ethical decisionmaking and performance of dentists. The aim of developing professional ethical codes for dentists is to provide an ethical standard for dentists' practice, to inform the public of these standards, and to achieve professional integrity [4]. The Universal Declaration of Human Rights emphasizes pn respecting the patients' dignity in health care [5]. Analysis of professional ethical responsibilities reveals a series of common irrefutable ethical principles; for example, there are no doubts about the necessity of respect, confidentiality, and fidelity. Although ethical considerations guarantee that these general principles are followed, they should be defined exclusively for relative parties. In professions such as dentistry where human relationships, with a patient or with a colleague, are stronger, the exact specification of these principles seems more necessary. Observation of patients' rights is one of the most important professional principles in medical ethics. Dental associations have developed codes of ethics. These codes are the written commitments arising from the implied contract between the dental profession and the society.

The most prestigious code is developed by the American Dental Association (ADA) under the title "Principles of Ethics and Code of Professional Conduct", which is updated annually. The latest version of the ADA ethical codes (2018) has three main components: "the Principles of Ethics, the Code of Professional Conduct, and the Advisory Opinions". Five fundamental principles are discussed in the ADA Principles of Ethics including patient autonomy, non-maleficence, beneficence, justice, and veracity [6]. In the United States, other dental associations such as, American Association of Dental Consultants, Texas Dental Association, California Dental Association, and American College of Dentists, have developed their codes of ethics, based on the ADA principles with the aim of promoting ethical behavior and maintaining the competence of individual members of the associations based upon standards of practice [7-10].

In Canada, the College of Dental Surgeons of British Columbia also developed their ethical codes based on ADA principles [11]. The Newfoundland and Labrador Dental Board and the Manitoba Dental Association have set up ethical codes based on the principles of autonomy, beneficence, non-maleficence, competence, and veracity and have determined ethical obligations toward patients, the public and the profession $[12,13]$. One of the most comprehensive ethical guidelines has been developed by the General Dental Council of England in 2013. This document regulates the standards of conduct, performance, and ethical principles and guidelines that govern dental professionals, which are applied to all members of the dental team as dental professionals. According to these standards, there are nine principles that dental professionals must always observe. For each principle, the patient expectations from the dental team have also been formulated [14]. In Australia, before applying the Health Practitioner's Regulation National Law in 
2010, dentists operated under different regulatory requirements in each state. Thus, each state and territory had their own ethical principles. The Australian Dental Association has published the Code of Conduct for registered health practitioners as the Dental Code of Conduct to guide the professional conduct of dentists in relation to the patient, the staff, the colleagues and the associations [15]. The Dental Ethics Manual was developed by the FDI World Dental Federation in 2007 and updated in 2018. In the previous version, ethical considerations in the dentists' relationship with their patients, society, and colleagues were discussed and in the last edition, ethical issues in 12 separate chapter were evaluated by providing clinical cases. A step-wise approach to ethical decision-making is helpful in this manual [16]. The Dental Council of India, in addition to determining the ethical duties and obligations of dentists, has also discussed unethical acts in the areas of soliciting, rebates and commission, running an open shop, doctor-patient sexual misconduct, and violation of the laws [17].

A great deal of attention has been recently paid to the development of the principles of medical ethics in Iran, resulting in the development of general and specific codes of ethics in the field of research, comprehensive documentation of the country's drug system, nursing code of ethics, professional ethics charter of psychiatry, and patients' rights charter $[1,19,20]$. In 2018, the Medical Council of the Islamic Republic of Iran published general ethical guidelines for healthcare professionals and affiliates under 11 titles. The Iranian medical community is required to observe the codes presented in the guidelines; failure to comply may result in prosecution and penalty [21]. These priorities are also shared in dentistry as a branch of medical sciences; however, due to the differences in the field of dental practice and thus patientdentist relationship compared with the medical field, it is imperative to develop specific ethical considerations for dentistry. Dentists have to make different decisions as a result of technological advances in prevention, diagnosis, and treatment as well as changes in the financial status and expectations of patients. The public release of ethical considerations will serve as a guide for service providers on how to deliver the service. Moreover, due to cultural, social and economic differences of Iran with other countries, certain issues in the dentist-patient relationship, such as unwillingness of some patients to be treated by a dentist of opposite sex according to religious beliefs and a greater proportion of out-of-pocket payment, are different from those in other countries. Since the establishment of a dental school with the management of Melczarski in 1930, no attempt has been made to develop codes of ethics for dentists despite their necessity [22]. Moreover, although professional dentists are committed to adhering to generally accepted ethical codes, few studies have evaluated whether dentists exercise these principles in practice. The aim of this study was to develop ethical codes for general dentists to serve as a preliminary source to achieve standardized codes, and to improve the dentists' professional performance in Iran.

\section{MATERIALS AND METHODS}

The present study was conducted at the Dentistry Research Institute of Tehran University of Medical Sciences, Tehran, Iran, from August 2016 to July 2018. This qualitative study was performed in three phases:

First Phase: A systematic search was conducted using published books, manuscripts, and articles as well as online databases to retrieve some guidelines and papers most relevant to the "code of conduct" and "code of ethics" in combination with the terms "dentist", "dentistry", and "dental".

Thirty ethical codes of dental foundations around the world and 56 papers on dental ethics were collected. Their records were analyzed through directed content analysis according to the method proposed by Zhang and Wildemuth [23] involving eight steps and finally, 196 items were extracted from the ethical codes and the articles. The items retrieved in this stage were used to develop the guidance questions used for the 
interviews. According to the conceptual framework extracted in the first phase, the qualitative data of the interviews were classified and codified.

Second Phase: Purposive sampling with maximum variation was applied to select the participants from the faculty members with different dental specialties. At the beginning of the interview, each participant was informed about the aim of the study and was ensured about the confidentiality of information. Informed consent was obtained from the participants. The guidance questions were used to conduct semi-structured interviews with dental specialists to assess their point of views about ethical considerations and priorities in dentistry and to identify the most important challenges and problems.

The interviews were continued until data saturation. Fifteen interviews were audiotaped, and the audio file of each interview was saved using the participant's unique code. It was immediately transcribed verbatim, and was analyzed according to the predetermined items. The statements made by the specialists served as a basis for the assembly of items. Overall, 105 items were obtained through the interviews, which were combined with the terms collected in the first phase. At the end of this phase, a preliminary draft of the codes of ethics, consisting of 196 items, was set up. The categories were deduced from the items using a continuous comparison method, and the items were placed in 12 proper subcategories according to their properties and similarities. The researchers continuously reviewed earlier stages of the research in detail. The content analysis approach was directed in this study. The conceptual framework of the results of previous studies and predefined classifications were used as the basis of directed content analysis and thus, the frameworks of existing theories were validated and developed [23].

Third Phase: In two expert panels with the participation of six experts who had adequate information and experience in this field, the preliminary draft of the codes of ethics was discussed and analyzed. Some items were deleted, some new items were added, and some items were combined. Eventually, codes were classified into five categories, as an initial schema, based on the perspectives and experiences of the experts in the sociocultural and religious context of Iran.

In order to maximize the validity and reliability of this qualitative study, the trustworthiness of the research was established according to Lincoln and Guba's evaluative criteria, including credibility, dependability, conformability, and transferability [24].

The credibility was established through prolonged engagement, persistent observation, external checks or peer debriefing, member checks, searching for disconfirming evidence and researcher credibility. The transferability or external validity was enhanced through thick descriptions of the site in which the studies are conducted. It was critical in allowing one to search for the similarities and differences between the situations. The dependability refers to data stability over time and over conditions. Researchers could enhance the reliability results by standardizing data collection techniques and protocols, documenting and inter-rater reliability (a consideration during the analysis phase of the research process). The audit trail was used to establish confirmability. The audit trail consisted of using an experienced person (with qualitative research background) to evaluate the degree of error of researchers and determine the accuracy and precision of the study process. Using triangulation for data collection was beneficial to promote the validity of the research. Data were collected from different sources such as literature as well as deep interviews aimed at reducing inherent bias associated with a single source. Different methods and theories have been used to collect data. Various researchers collected, interpreted, and analyzed the data [24]. Finally, the first edition of the codes of ethics was prepared, which comprised of 90 codes in five categories. In sessions with three experts in the field of writing ethical codes, the codes were extracted and finalized as the final edition of the ethical codes. A questionnaire 
with 5-point Likert scale was designed, and 20 dentists from the target group with more than 15 years of experience were invited to comment on the clarity of the items to assess the face validity of the codes; the necessary changes were made accordingly. The ethics of the study was approved by the ViceChancellor in Research Affairs of Tehran University of Medical Sciences (IR.TUMS.VCR.REC.1395.732). The objectives of the study were explained to the participants and their consent was obtained. The information was recorded anonymously and securely stored.

\section{RESULTS}

In this study, 56 articles, 30 ethical considerations and 15 interviews were reviewed. According to the subjects mentioned in one document or more, duplicate and similar codes were merged. Ultimately, 196 items were categorized into 12 categories based on their concepts. During two expert panels, the larger number of ethical codes were finalized to 90 codes and classified into five principles. Twenty dentists (12 women and 8 men) with a mean age of 40 years and an average experience of 15 years participated in evaluation of the face validity of the ethical codes and commented on the clarity of items (Table 1). Analysis of the handwritten notes of participants resulted in text correction of 8 original codes.

Table 1: Clarity of each item of ethical codes

\begin{tabular}{ll}
$\begin{array}{l}\text { 5-point Likert scale } \\
\text { of questionnaire }\end{array}$ & Number (percentage) \\
Very high & $54(30 \%)$ \\
High & $89(49.4 \%)$ \\
Moderate & $15(8.3 \%)$ \\
Bad & $10(5.5 \%)$ \\
Very bad & --- \\
\hline No answer & $12(6.7 \%)$ \\
\hline
\end{tabular}

The results of the present study comprised a set of trial codes that could be employed as a basis for developing ethical codes for Iranian dentists in collaboration with all representatives and policy-makers as shown in Table 2 .

\section{DISCUSSION}

A review of the code of ethics for dentists developed by different dental associations in different countries reveals their commonalities and similarities; therefore, they can be used as a basis for developing ethical codes. The basis of the most designed codes is the codes established by the ADA [6], American College of Dentists [10], General Dental Council (GDC) [14], and FDI World Dental Federation [16]. These codes have been reviewed several times. However, the basis of the ethical codes developed in this study cannot be anything beyond the common codes in other countries. Cultural, social and economic differences between Iran and other countries underline the importance of developing a set of ethical codes for Iranian dentists. The majority of the codes were related to the dentist-patient relationship due to its importance. The main principles that were developed included considering and maintaining the patients' interest as a priority, respect for human dignity and patient autonomy, confidentiality of patient information, the excellence of knowledge and skills, and building trust.

Three or four relative parties are defined for dentists in the FDI manual [16] namely the Code of Ethics of the Newfoundland and Labrador Dental Board [12], the Manitoba Dental Association [13], Australian Dental Association [15] and Dental Council of India [17]. The obligations and duties of Dentists towards patients, community, colleagues and their profession were specified. The ethical aspects of the dentist-patient relationship focused on patients' benefits, well-being, communication, consent, veracity, confidentiality and timely referral. All these items were considered in the present study. In terms of relationship with patients, two interesting issues in the code were emphasized. One of them is the subject of the patient's complaint and how to deal with it, which is addressed in the GDC code. Code 1-8 of the list developed in the present study was written in this regard. 
Table 2: Codes of ethics

\section{Principle 1: Considering the patients' interest as a priority}

1-1 Professional dentists always consider the principles of beneficence and non-maleficence for patients.

1-2 Professional dentists should not practice when their abilities are compromised for any reason, such as old age, disease, fatigue, drug use, mental health problems, etc.

1-3 Professional dentists are obligated to consider patients' interests, i.e., their health and well-being. When patients' interests conflict with that of patients' companions, other colleagues or the dentist, patients' interests should be considered as a priority.

1-4 Professional dentists shall not accept a formal or implicit contract that violates patients' interests.

1-5 Professional dentists provide proper feedback to the related authorities if they encounter organizational regulations contradicting patients' rights and interests.

1-6 Professional dentists practice according to their professional knowledge and expertise and refer the patients if they lack the required skills.

1-7 Professional dentists are required to have an individual medical record for each patient in accordance with the record standards, to document all diagnostic and therapeutic stages in the record in an intelligible and understandable manner for colleagues, and to facilitate patient access to this information.

1-8 Professional dentists respect the patients' right to complain and respond to their complaints in a responsible manner.

1-9 Professional dentists use their best efforts to promote patients' health.

1-10 Professional dentists are held accountable for their professional responsibilities.

1-11 Professional dentists accept the responsibility for their medical errors and take measures to compensate for the error to satisfy the patient.

1-12 Professional dentists have a holistic view of the patient and always consider all aspects of patients' health and well-being.

1-13 Professional dentists keep the practice environment clean and safe.

1-14 If professional dentists are certain that the people visiting their office or other referral healthcare centers are at risk of injury for any reason (shortcomings in disinfection and sterilization of the practice environment and equipment, infectious diseases of the colleagues and staff, or their unprofessional conduct), they will use their best efforts to resolve the issues, and if necessary, they report the matter to the relevant authorities.

1-15 Professional dentists communicate with parents or guardians, remind them of necessary cares, and guide them if they notice any shortcoming or flaw in the oral and dental care of a child or a vulnerable person.

1-16 If a professional dentist suspects child abuse, he/she will act in accordance with legal rules and make every effort to support the child.

1-17 Professional dentists guide patients based on prioritizing their benefits, avoid misleading phrases and statements, and never offer an inappropriate treatment.

1-18 Professional dentists adjust the patient's treatment plan according to the patient's limitations, characteristics, individual differences, and tolerance. They should not make their patients tired or uncomfortable.

1-19 Professional dentists should not take advantage of seemingly altruistic advertisements to attract more patients.

1-20 Professional dentists should not issue false medical certificates, including sick notes or certificates required for reimbursement of medical bills.

1-21 Professional dentists avoid unnecessary service and futile treatments.

1-22 Professional dentists use high-quality and standardized materials, products, and instruments approved by the relevant authorities. 
Table 2 cont'd

\section{Principle 2: Respect for human dignity and patient autonomy}

2-1 Professional dentists design their office and waiting room appropriately considering the patients health and comfort.

2-2 Professional dentists consider courtesy and respect human dignity in treating all people, including colleagues, staff, students, patients, and companions.

2-3 Professional dentists should not discriminate in communication with patients based on sex, age, ethnicity, race, nationality, health, disability, lifestyle, or any other irrelevant factor.

2-4-1: The patient does not respect the country's laws and regulations, including: Not observing Islamic cover

Professional dentists visit all patients with ethical manners and only refrain from visiting

2-4 non-emergency cases if it does not deprive the patient of receiving the required service, such as one of the following conditions: and religious considerations or alcohol consumption.

2-4-2: The patient does not accept the approved fees. 2-4-3: The patient is impolite and over-expectant.

2-4-4: The refusal of the patient is not associated with discrimination.

2-4-5: The refusal of the patient is not associated with disrespecting or insulting the patient.

2-4-6: The non-acceptance cases are informed in advance.

2-5 Professional dentists have respect for patients and are polite in all exposures and conversations, whether in the presence or absence of the patient.

Professional dentists have a good and respectful relationship with the patients' companions and provide them with a suitable environment.

Professional dentists allow the patients to be accompanied by a companion if they are incompetent in decision-making.

Regarding patients with decision-making competency, professional dentists allow the presence of a companion if the following conditions are met:
2-8-1: The patient consents to the presence of a companion.

2-8-2: The dentist believes that the presence of a companion is in the patient's best interest.

2-8-3: The companion does not interfere with the dental procedure.

2-8-4: The presence of the companion does not pose a health risk.

2-9 For patients incapable of self-care, professional dentists consider the patients' companions and families as part of the treatment team and share the necessary information with them.

2-10 Professional dentists shall not accept a contract that would violate the patient's respect.

2-11 Professional dentists value the patients' time and visit them according to the appointment schedule.

2-12 Professional dentists politely inform scheduled patients if they are forced to give priority to urgent cases.

2-13 Professional dentists should not start the treatment without obtaining the patients' or their legal guardian's informed consent, except for urgent cases.

2-14 Professional dentists should not avoid treating patients with bloodborne pathogens and should practice in a polite manner and with good behavior according to scientific principles. 


\section{Table 2 cont'd}

2-15 Professional dentists respect the patient's beliefs and culture of different ethnic Iranian groups.

2-16

Professional dentists are aware of communication skills and make every effort to establish effective communication with their patients.

Professional dentists consider the followings to

2-17 respect the patient's choice and to promote shared decision-making:
2-17-1: Listen to the patient's words and concerns carefully and with empathy.

2-17-2: Inform patients of the overall status of their oral and dental health.

2-17-3: Share the information required for decision-making with the patient.

2-17-4: At the request of the patient or if this has any impact on decision-making, inform the patient of the type and the material of the consumables, instruments, and equipment.

2-17-5: Professional dentists should inform the patient of treatment costs in detail.

2-17-6: Explain the advantages and disadvantages of different treatment choices in a simple and understandable language.

2-17-7: Answer the patient's questions completely, accurately, and in an intelligible manner.

2-17-8: Professional dentists should not force the patient to make decisions and should give the patient enough time for decision-making.

2-17-9: Professional dentists make the final decision based on the patient's benefit while considering the patient's preferences as well.

2-17-10: Professional dentists ask for the presence of an interpreter if the patient does not speak the same language as them.

\section{Principle 3: Confidentiality of patients' information}

Professional dentists keep the relevant and unrelated information about the patient's treatment, in

3-1 writing or otherwise, confidential at all times, refrain from disclosing it, only use it for treatment purposes; any other use of this information is subject to permission from the patient or judicial orders.

3-2 Professional dentists shall not accept a contract requiring disclosure of patient information (such as insurance research, advertising contracts, etc.) unless the patient becomes aware and declares his/her consent.

3-3 Professional dentists should not disclose any patient information on the Internet that could lead to identifying the patient directly or indirectly.

3-4 Professional dentists make sure the patient's identity remains anonymous if they use the information for online education.

3-5 Professional dentists safeguard the patients' medical records and prevent any unauthorized access to them.

3-6 When professional dentists are required by law or judicial orders to share patient information, they should only disclose that particular case to the least necessary extent.

3-7 Professional dentists consult the relevant author 


\section{Table 2 cont'd}

During medical consultation, professional dentists ask for permission from the patient to disclose

3-8 information. If the patient refuses to consent, they avoid consultation and either continue treatment or refer the patient to another center at their own discretion.

\section{Principle 4: The excellence of knowledge and skills}

4-1

Professional dentists review their own weak and strong points constantly and make their best efforts to have a realistic perspective in this regard.

4-2 Professional dentists always try to update their knowledge, skills, and performance in line with their professional education.

4-3 Professional dentists are serious about continuous learning of standard treatment protocols and seek advice and guidance from trusted sources.

4-4 Professional dentists use valid references to obtain information about treatment methods, devices, and materials and ignore non-scientific and invalid sources.

4-5 Professional dentists share their knowledge, skills, and experiences with other colleagues and healthcare professionals to maintain and promote the status of dentistry as a profession.

4-6 Professional dentists carefully read the regulations and by-laws released by the Ministry of Health and Medical Education, are fully aware of them, and apply them in practice.

4-7 Professional dentists constantly improve their physical and mental capabilities to enhance their professional performance.

4-8 Professional dentists sincerely help and guide colleagues if they have the required knowledge and skills.

\section{Principle 5: Building trust}

5-1 Professional dentists always consider building trust as a top priority in all professional relationships.

5-2 Professional dentists adhere to the "Patients' Rights Charter in Iran" and place it where patients/clients can see it.

5-3 Professional dentists have a fair and honest performance towards patients, society, and their colleagues.

5-4 Professional dentists cooperate with other members of the therapeutic team to deliver excellent service to patients.

5-5-1: Avoid judging the social, practical, and scientific personality of that colleague.

With regard to patients' therapeutic interest as a

5-5-2: Do not make unethical judgments about other top priority, professional dentists consider the colleagues and do not criticize their former service.

5-5 top priority, professional dentists consider the treated by a colleague:

5-5-3: Make a fair and clear comment on the current status and condition of the patient.

5-5-4: Praise the effective and correct practice of that colleague.

Professional dentists politely give appropriate feedback to colleagues who have improper professional

5-6 conduct in order to promote professional behavior and enhance patients' trust by paying attention to their treatment preferences.

Professional dentists report to the relevant authorities upon encountering colleagues who continue

5-7 faulty professional conduct (ethical or clinical) and undermine the public trust regarding professional dentistry despite receiving appropriate feedback.

5-8 Professional dentists shall not accept a contract that leads to the violation of patients' rights or disrupts the public trust regarding professional dentistry.

5-9 Professional dentists should not deceive patients regarding the extent and severity of the disease and should not indulge their patients in false hopes and guarantee of treatment. 


\section{Table 2 cont'd}

5-10 Professional dentists provide true and fair explanations about treatment options and the materials, instruments, and equipment being used, and avoid unrealistic and false advertising.

When a patient is referred to professional dentists for consultation or seeking their expert opinion, they

5-11 return the patient to the referring dentist along with a written report and should not embark on treatment without the patient's consent and approval of the former dentist.

When a patient is referred to professional dentists for certain treatments and they found that further

5-12 treatment is required, they make a written report to the referring dentist and ask the patient to consult with the former dentist and return for treatment if he/she wishes to do so.

5-13 Professional dentists seek the approval of the referring dentist when a patient is referred to them for consultation and they realize that it is necessary to seek the professional opinion of other colleagues.

5-14-1: Communicate with patients in a respectful manner.

5-14-2: Do not disclose patients' information.

5-14-3: Maintain order and cleanliness.

Professional dentists select their staff among experienced and reliable people, accept responsibility for their actions, and are held accountable for their behavior. They supervise all auxiliary personnel to consider the followings in their contact with clients:

5-14-4: Do not make unnecessary recommendations to patients/clients unless specifically asked for by the employer.

5-14-5: Do not make comments and prejudgments on the service provided by the employer and other dentists. 5-14-6: Refrain from accepting unconventional gifts that may discriminate among patients.

5-14-7: Do not discriminate between patients.

5-14-8: Respect the patients' time.

5-15

Professional dentists consider and adhere to scientific principles and health recommendations to prevent the transmission of bloodborne pathogens.

5-16 If the staff is infected with blo contact with patients.

5-17 Professional dentists should not discontinue the treatment of a patient unless:

5-17-1: Patient abandonment is at the request of the patient, in which case, they provide the necessary guidance regarding the consequences of their decision, explain how to prevent further damage, and obtain their written consent.

5-17-2: If patient abandonment is because of the dentist's scientific and skill incompetency, the patient should be referred to competent colleagues for treatment.

5-17-3: Patient abandonment is because of the patients' non-adherence to ethical and legal regulations or financial commitments. Nevertheless, abandonment should not jeopardize the patient's health. In this case, appropriate health centers are introduced to the patient.

Professional dentists should not establish any non-professional relationship with patients irrelevant to

5-18 the treatment process (including romantic, sexual, and occupational relationships as well as social friendship) that might affect their professional judgment and treatment performance. 


\section{Table 2 cont'd}

5-19 Professional dentists are allowed to start a romantic relationship with a former patient whose treatment has been completely finished.

5-20 Professional dentists accept responsibility for dental laboratory technicians' errors, offer their apologies to the patient, and repeat the process of treatment free of charge.

Upon encountering medical errors by other members of the therapeutic team, professional dentists

5-21 offer their apologies to the patient, make a report of the case to the related colleague, and request a free of charge and out of turn correction of the error.

5-22 If professional dentists make a mistake during teamwork, they welcome remarks from colleagues and accept responsibility by offering either free of charge treatment or reimbursement

5-23-1: Professional dentists avoid any judgment and leave the matter to the relevant authorities. If required, they should present their expert opinion to the authorities in accordance with regulations in

In dealing with a patient due to the error of other dentists: an honest and responsible manner.

5-23-2: Professional dentists explain the current problem and treatment solutions clearly in light of objective evidence and should not comment on the dental practice of other colleagues.

5-24 Professional dentists consider honesty, responsibility, and rectitude when presenting as testimony and witness to uphold justice if they are summoned to appear before a court of law or do so at the request of a patient.

5-25 Upon encountering any occurrence of an adverse reaction to the materials and instruments used, professional dentists report the matter to the relevant authorities.

Professional dentists should not participate in the direct sale and marketing of dentistry products. However, if they know by experience that some products are more useful and some are harmful or useless, they can recommend using or not using them by considering the best interest of patients, provided that they have no financial gains.

5-27 Professional dentists should not make non-scientific and non-evidence-based decisions.

5-28 Professional dentists should not present non-scientific and non-evidence-based opinions in the media.

5-29 Professional dentists should not cooperate with the media to gain fame and popularity.

5-30 Professional dentists place their medical license and field of specialty where patients can see them and should not deceive patients with inauthentic licenses and unwarranted certificates.

5-31 Professional dentists should not use commercials and advertisements to build trust and attract patients. They should consider profit-driven marketing below their professional dignity and avoid it.

5-32 Professional dentists should not accept gifts from patients or pharmaceutical companies because this might influence their professional judgment. They should politely apologize to the donor.

5-33 Professional dentists should not accept rewards and incentives (including remuneration, gifts, discounts, and patient cross-referral) for patient referral and should avoid offering such rewards and incentives as well.

5-34 Professional dentists refer patients by considering patients' best interest, scientific evidence, and the capabilities of the referral center and never pursue their own personal benefits and interests.

Professional dentists cooperate with pharmaceutical companies and industrial centers related to the field of dentistry (including all scientific, research, administrative, economic, commercial, marketing, and product promotion collaborations) only if they are certain about their ethical and scientific competence and believe that this cooperation is in the best interest of patients. 
Another point of interest was the interaction with uncooperative patients [16], which was also referred to in the Code 2-4 of this study. According to this code, an impolite and over expectant patient who causes an inconsistency is addressed as part of the condition for refusal to be accepted by the dentist. Some issues, such as attention to the patient's financial limitations and waiver of remuneration have been addressed in some guidelines [16]. These topics can be categorized as meta-ethics and were not therefore addressed in this draft. Patients' financial limitations, are raised in the code of FDI [16] that are not addressed in this study. In some certain cases, dental clinicians may refuse to receive a fee for service from patients who cannot afford the cost of dental treatments. Since it would be considered a charity act and categorized under the category of meta-ethics, we did not address it in this draft.

In dentist-society relationship, the guidelines addressed some topics including dual loyalty $[8,16,17]$, resource allocation [16], public health $[6,8,14,16]$, and contracts and third parties $[9,12,13]$. The subject of "building trust" in principle 5 has been emphasized in the present study and it should be a top priority in all aspects of practice; therefore, loyalty to society was also considered in the present study. It was decided to add broader social aspects to the subsequent revisions of the code because it is the first time that Iranian dental clinicians face these ethical codes. Providing ethical codes of conduct for a specific medical specialty does not mean that there had not been any ethical perspective or culture available in that field beforehand. Obtaining a peer reviewed and widely accepted list of ethical code may help improving dentists' awareness, knowledge and behavior and finding answers for some confusing situations. Prioritizing the patient's interests to the third parties can be done in accordance to the contracts. This topic has been extensively studied in various sections of this study. For example, Codes 1-4, 2-10, 3-2, 5-8 have discussed ethical obligations for dentists. They should refuse to accept the contracts that would violate the patients' interests and also, respect for human dignity, patients' confidentiality and public trust. Even though paying attention to patients' oral and dental health is one of the basic professional responsibilities of dentists, topics such as resource allocation and noticing public health policies were not considered as a moral obligation for dentists in the present list of codes because the cost of dental treatments in Iran is mainly paid out-of-pocket. Relationship of dentist colleagues have several ethical topics such as, ethical judgment regarding the past performance of colleagues, reporting unsafe and unethical practice, and constructive and helpful communication with other health professionals that were addressed in some guidelines [6,13-16]. All members of the dental team are involved in shaping the patients' experience of dental treatment and public trust to this profession. Therefore, professionals should help each other in order to provide the best care for patients and prioritize their interests. Because of importance of the professional relationship between colleagues and other health professionals, dentists need to share their knowledge, skills and experiences which has been emphasized in Code 4-5 of the present study. Unethical judgments about colleagues' performance was discussed in Codes 5-5-2 and 5-23-1. Codes 5-7 and 1-14 point to the importance of reporting inappropriate professional performance and risk of injury. The fields of education and research are beyond the scope of the present study and this paper did not intend to address the ethical issues associated with these topics.

In responsibilities to the profession, there are various ethical issues such as self-regulation in the profession [12,13], advertising [6,1013,17] and obligation towards professional associations, which were mentioned in various codes. The self-regulation topic that would lead to public trust was discussed in Codes 5-7 and 5-23 of the present study. The issue of how to deal with the mistakes of other dentists and reporting the unsafe and unethical practice to relevant authorities, and the related requirements in this respect were also mentioned. Our designed codes do not 
address advertising because it should comply with the regulations of the Medical Council of the Islamic Republic of Iran [21]. In the present study, the greatest emphasis was made on the dentist-patient relationship. As the supervisory role of related dental associations on the ethical performance of dental professionals is not firm enough in Iran, this study did not enter the field of professional relationships with the related councils and associations. The codes obtained in this study were all in line with the five fundamental principles of autonomy, nonmaleficence, beneficence, justice, and veracity. In 2016, Lovino [25] criticized the ADA codes and suggested adding the principle of "respect for human dignity" as the sixth principle to the ADA Principles of Ethics and Code of Professional Conduct and expressed its importance in "the foundation of a dentist's professional obligations in a new era of genetics in dental research, education, and practice". In the second principle of the present study, the topic of respecting human dignity has been discussed specifically in detail.

One of the most important issues which has been mentioned in some guidelines is the consultation, referral and testimony of the dentist as a witness or expert [6,9]. Due to the importance and significance of these topics, consultation with experts and timely referral of patients have been discussed in detail in Codes 1-6, 5-17-2 and 5-34 in this study. The Code 5-24 addresses the issue of testimony at the time of summoning a judicial authority or at patient's request and reaffirms the principles of veracity, integrity and responsibility. Fee splitting is also one of the tempting propositions to be dealt in dental practice [6,17]. The Code 5-33 emphasizes on dentists refusing the offers, rewards or incentives for patient referrals. In the era of globalization and explosion of information, the benefits and disadvantages of social media are a hot topic for all users. The codes of ADA and GDC and also the Handbook of American College of Dentists considered specific codes about sharing any information or comments about patients on social networking or blogging sites that could affect the public trust $[6,10,14]$. In the present study, there is no independent code referring to this issue, but it has been considered implicitly in the principle of confidentiality of patients' information.

In design and development of these codes, we had to choose the notions from all ethical concepts that brought about observable or evaluable criterions which governed behavior or activity to be performed or abandoned. Hence, we could not convert many of the ethical virtues which were especially mentioned in the religious context into ethical codes.

Although many of the central virtues of Islam, such as piety, purity and faith have a significant impact on apparent behaviors, they are not directly measurable by others. Nevertheless, the ethical codes for Iranian dentists were drafted considering Islamic values and beliefs as well as the dominate culture, and the authors paid special attention to the abovementioned items in all stages. It seems that the codes developed in this study are similar to codes developed in Western and secular societies but an overall result can be deduced that Islamic principles are very similar to the ethical principles commonly accepted by other ethical schools and scholars, and the conceptual foundations of Islam are not different from moral propositions. Therefore, although none of the codes of this draft has a direct reference to Islamic principles, the spirit of Islamic teachings including honesty, truthfulness, confidentiality and fidelity is considered in all codes. As a result, the codes developed in this study are very similar to codes released by other associations $[7,9,11,14,15]$. In addition to the similarities that exist between the codes of the present study and other associations, our guideline also includes other codes exclusively developed for the cultural atmosphere of Iran.

For example, Code 5-5 discusses interaction with patients previously treated by other dentists, and Codes 5-11 and 5-12 address the principles of consultation and continuing the therapeutic relation with such patients, which are the codes influenced by the Iranian 
culture. Moreover, communication skills and empathic listening were addressed under Codes 2-16 and 2-17; whereas, these items have not been considered in codes developed and released by other associations. In addition, in this study, several new codes were obtained through interviews, which were not addressed in other associations. These codes are listed as follows:

- $\quad$ Code 2.1, which addresses design of the office and waiting room

- $\quad$ Code 2.8, which addresses conditions for the presence of patient's companions at his/her bedside.

- $\quad$ Code 3-8, which addresses conditions for the disclosure of patient's information during counseling

- Code 4-7, which addresses the improvement of dentist's physical and mental capabilities.

- Code 5-2, which addresses the "Patients' Rights Charter"

- $\quad$ Code 5-5-4, which addresses "praise the effective and correct performance of colleagues

- $\quad$ Code 5-14, which addresses the ethical requirements for the office staff.

These ethical codes can be considered as the innovation of this study, which were developed based on the specific type of interactions of individuals at different levels. This principle was designed on the basis of certain goals and in the specific cultural context of the country. For example, there is no dental referral system in our country and the referral for counseling is mainly based on the personal relationships of the dentist. Therefore, code 3-8 concerned the disclosure of patients' information, which is not seen in the codes of other associations in this extent. The Codes 4-7, 2-1 and 5-5-4 were obtained precisely in a deep personal interview. Because of the particular culture of the Iranian families, family members have an important role in decision making of each other. Hence, the codes related to the presence of patient's companion were emphasized in the focus groups [26].

The Code 2-8 was also emphasized by the expert panels. Lack of knowledge and communication skills of the auxiliary personnel leads to misinterpretations that allow them to comment and sometimes disrespect patients; thus, the Code 5-14 was developed for the office personnel. In qualitative studies, all stakeholders should be involved in order to obtain inclusive results compatible with our culture and religion. We could not find a religious representative from the main religion centers. We tried to compensate for this major limitation by inviting people with a religious background to the panels. It was tried to use a proper tone in all ethical codes. Some ethical issues, such as advocacy of the society and meta-ethical issues, may cause resistance in dentists due to their complexities, and hence, they will be addressed in subsequent revisions. In summary, it seems that according to these codes, the first professional responsibility of dentists is to pay attention to the patients' needs and to provide an environment where there is respect for the values, beliefs, rights, and human dignity. Ethical codes lay down the behavioral regulations and ethical considerations of a profession, which all members of the profession shall be bound to observe in their practice. Ethical codes also serve as criteria for making ethical decisions in complex and sensitive situations in order to establish an appropriate relationship between dentists and patients, patients' companions, and other dentists.

\section{CONCLUSION}

The difference in the level of knowledge of dentists and patients regarding dental science necessitates this profession to have special ethical considerations. Dental clinicians must be aware of their ethical responsibilities since they face ethical challenges in their daily work. In this way, dentists will be able to design a moral framework for difficult decisions. We provided a draft of code of ethics for the Iranian dental clinicians. These codes are intended to inform dentists about their roles and responsibilities. A set of values were articulated in this ethical code draft. The 
patient's interest is best protected when decisions reflect the values of ethics rather than business purposes. An important goal of these codes is to consider the values that drive human dignity. These codes are expected to provide a practical guidance to help dentists solve ethical issues. There may be barriers to fulfill the ethical requirements by organizations or stake-holders. A national consensus for implementation of ethical codes is the first step in ethical practice, and further efforts in continuing dental education programs are recommended to enhance the knowledge related to clinical ethics. We look forward to share our document to hear feedbacks from dentists and dental policymakers. These codes can be further analyzed and finalized by dental policy-makers and authorities as a commitment document for dental professionals to take steps towards building trust and promoting patients' welfare and satisfaction.

\section{ACKNOWLEDGMENTS}

This paper was part of a thesis submitted by the first author for a PhD degree in medical ethics and was funded by the Dentistry Research Institute, Tehran University of Medical Sciences. The authors are grateful to all the dentists for their valuable participation in this study.

\section{CONFLICT OF INTEREST STATEMENT}

The authors declare that there is no conflict of interests regarding the publication of this paper.

\section{REFERENCES}

1. Parsapoor A, Bagheri A, Larijani B. Patient's rights charter in Iran. Acta Med Iran. 2014;52(1):24-8.

2. Newsome PR, Langley PP. Professionalism, then and now. Br Dent J. 2014 May;216(9):497-502.

3. Jones AR, Siegler M, Winslade WJ. Clinical ethics. A practical approach to ethical decision in clinical medicine. 8 $^{\text {th }}$ ed., McGrawHill Education, New York, 2015:1-2.

4. Edwards AA. An important change to the American Dental Association Principles of Ethics and Code of Professional Conduct: The evolution of Section 5.H, and a new perspective on the announcement of specialties. J Am Dent Assoc. 2017 Feb;148(2):125-7.

5. United Nations. Universal Declaration of Human Rights. 1948; Available at: https://www.un.org/en/universaldeclaration-human-rights/

6. American Dental Association. The ADA Principles of Ethics and Code of Conduct. 2018; Available at: https://www.ada.org/en/about-theada/principles-of-ethics-code-ofprofessional-conduct

7. The American Association of Dental Consultants. Code of Ethics. 2018; Available at: https://www.aadc.org/site page.cfm?pk asso ciation webpage menu $=4398 \&$ pk association webpage $=9748$

8. Texas Dental Association. TDA Principles of Ethics and Code of Professional Conduct 2015; Available at: https://www.tda.org/Portals/0/manuals/TD A\%20Revised\%20CODE\%20of\%20Ethics $\% 2$ 05-14-14.pdf

9. California Dental Association. Code of Ethics. 2017; Available at: https://www.cda.org/Portals/0/pdfs/code o f ethics/code of ethics.pdf

10. American College of Dentists. Ethics Handbook for Dentists. Gaithersburg, Maryland, 2016:1-23.

11. College of Dental Surgeons of British Columbia. Code of ethics 2015; Available at: https://www.cdsbc.org/CDSBCPublicLibrary Code-of-Ethics.pdf

12. Newfoundland \& Labrador Dental Board. Code of ethics. 2008; Available at: http://nldb.ca/downloads/Code-ofEthics.pdf.

13. Manitoba Dntal Association. Code of ethics 2002; Available at: https://www.manitobadentist.ca/profession al-legislation-ethics.cfm.

14. General Dental Council. Standards for the Dental Team. 2013; Available at: https://standards.gdc-uk.org

15. Australian Dental Association. Code of Ethics and Disciplinary Procedures. 2012; Available 
https://www.adansw.com.au/getattachment LAbout/Public-Constitution,-Policies-and-ByLaws/Code-of-Ethics-and-DisciplinaryProceduresAug2012.pdf.aspx

16. World Dental Federation. Dental Ethics Manual 2. 2018; Available at: https://www.fdiworlddental.org/sites/defaul t/files/media/resources/fdidental ethics manual 2.pdf

17. Dental Council of India. Code of Dental Ethics. 2014; Available at: http://www.wbja.nic.in/wbja adm/files/The \%20Revised $\% 20$ Dentist $\% 20$ (Code $\% 20$ od $\% 2$ 0Ethics)\%20Regulations,\%202014.pdf

18. Ammari M, Ghoddousi A. Codification of the psychiatric patients rights charter in Iran. J Fundam Mental Health 2018 NovDec;20(6):382-94.

19. Sanjari M, Zahedi F, Aalaa M, Peimani M, Parsapoor A, Cheraghi MA, et al. Code of ethics for Iranian nurses. J Med Ethics Hist Med. 2011 Dec;5(1):17-28.

20. Medical Ethics and History of Medicine. Code of ethics for the national pharmaceutical system. Available at: https://pec.kums.ac.ir/kums content/media/ image/2016/07/85246 orig.pdf

21. Islamic Repiblic of Iran Medical
Council. Professional Ethics Guide for Medical Professionals. 2018; Available at: https://centlab.tbzmed.ac.ir/Uploads/User/ 5460/files/\%D8\%A7\%D8\%AE\%D9\%84\%D 8\%A7\%D9\%82 \%D9\%BE\%D8\%B2\%D8\%B 4\%DA\%A9\%DB\%8C1 83170.pdf

22. Schulein TM. A chronology of dental education in the United States. J Hist Dent. 2004 Nov;52(3):97-108.

23. Qualitative analysis of content by Zhang Y, Wildemuth BM. Available at: https://pdfs.semanticscholar.org/b269/343 ab82ba8b7a343b893815a0bae6472fcca.pdf. 24. Lincoln YS, Guba EG. Criteria for Assessing Naturalistic Inquiries as Reports.1988 Available at: https://eric.ed.gov/?id=ED297007

25. Lovino R. Revising the American Dental Association Principles of Ethics and Code of Professional Conduct: Adding "Respect for Human Dignity" as the sixth principle of dental ethics to accommodate advances in genetic science. J Am Dent Assoc. 2016 Dec;147(12):918-22.

26. Rahimi SA, Alizadeh $\mathrm{M}$, Légaré $\mathrm{F}$. Shared decision making in Iran: Current and future trends. Z Evid Fortbild Qual Gesundhwes. 2017 Jun;123:52-5. 